\title{
Joint Power and Beamwidth Optimization for Full Duplex Millimeter Wave Indoor Wireless Systems
}

\author{
Akhtar Saeed \\ Faculty of Engineering and Natural Sciences \\ Sabanci University \\ Istanbul, Turkey 34956 \\ akhtarsaeed@sabanciuniv.edu
}

\author{
Ozgur Gurbuz \\ Faculty of Engineering and Natural Sciences \\ Sabanci University \\ Istanbul, Turkey 34956 \\ ogurbuz@sabanciuniv.edu
}

\begin{abstract}
In this paper, a joint power and beam-level beamwidth control scheme is proposed for full duplex (FD) millimeter wave (mmWave) indoor wireless systems. Energy efficiency of the proposed scheme is investigated considering various system parameters, such as maximum transmit power level, level of self-interference cancellation and pilot transmission overhead. With this analysis for a realistic indoor wireless communication scenario, the feasibility of FD is studied for mmWave links, considering their specific propagation characteristics, namely, narrow transmission and reception beam-level beamwidths and high absorption losses, as well as massive bandwidth which is much larger than the existing sub $6 \mathrm{GHz}$ bands. We evaluate the performance of the proposed FD mmWave system for three power budget schemes (low, moderate and high) in terms of average total energy efficiency. Our simulation results show that, for currently available state-of-the-art self-interference cancellation levels, FD mmWave with proposed joint power and beam-level beamwidth control outperforms the smart half duplex (HD) mmWave with joint transmission slot and beam-level beamwidth control by a factor of up to four times and improves FD mmWave with only power control by up to $33.92 \%$. If higher (close to ideal) selfinterference cancellation can be achieved, the net average total energy efficiency improvements over existing abovementioned schemes, are up to 4.8 times and $26.45 \%$, respectively. It is concluded that with the proposed joint power and beamwidth control, the current FD mmWave technology promises a good potential for indoor wireless networks.
\end{abstract}

Index Terms- energy efficiency, full duplex communication, millimeter wave, self-interference

\section{INTRODUCTION}

The ever increasing demand for higher data rates in wireless communication networks has made it inevitable for the telecommunication policy makers and the researchers to look for possible bands other than the existing sub $6 \mathrm{GHz}$, which is almost saturated [1]. Millimeter wave (mmWave) communication $(28,60,73 \mathrm{GHz})$ has recently proved to be one of the candidates for meeting this demand, owing to its massive bandwidth [2]. Due to very high operating frequency, mmWave communication exhibits certain unique characteristics, such as high antenna directivity gains and high absorption losses [3]. mmWave communication is also a preferable candidate for indoor wireless communications due its to low cost as compared to wired access points [4]. Several standards have been developed for short-range mmWave communications, such as IEEE $802.15 .3 \mathrm{c}$ for wireless personal area networks
(WPANs) [5] and IEEE 802.11ad wireless local area networks (WLANs) [6]. mmWave communication has evolved as an ideal solution to the future $5 \mathrm{G}$ green communications as they are cost and energy efficient [7], while concurrently offering massive data rates for the applications, such as live-streaming multimedia traffic [8].

Wireless communication has traditionally been implemented in half duplex (HD) mode as it is not possible to transmit and receive simultaneously in the same band. Recent works have shown that with in-band full duplex (FD) communication, time and frequency resources can be utilized at the same time and frequency, promising doubled capacity. This is achieved at the expense of self-interference (SI) cancellation, which introduces extra complexity and power consumption for FD radios [9]. Successful and sufficient mitigation of the SI has been demonstrated on FD radio prototypes in current wireless systems [10], as well as mmWave radios [11].

FD communication relies on SI cancellation at different levels, such as passive suppression (PS) [12], analog SI cancellation (ASIC) [13] and digital SI cancellation (DSIC) [10]. Except for PS, the SI cancellation techniques are power consuming, posing additional power overhead for the radio. Power or energy efficiency is crucial for mmWave communication systems as the global wireless communication is transitioning towards the green communication paradigm with $5 \mathrm{G}$, aiming to be throughput as well as energy efficient [14]. Energy efficiency is stipulated as the ratio of the achievable transmission rate to the consumed power [14].

mmWave technology is attractive for indoor wireless communications due to high data rates within small range [15]. In [16], a joint beam-level beamwidth and power control is proposed for maximizing the throughput of a HD mmWave based network, highlighting the benefits and importance of optimizing beam-level beamwidths of millimeter wave communication links. With the implementation of FD in mmWave radios [11], research on FD mmWave is gaining more interest for enhanced rates [17]. In [7], an overview of energy efficiency in FD mmWave relaying networks is provided along with an in-depth overview of a power consumption model. In [18], FD mmWave relaying with transmit power control is proposed; however optimization of beam-level beamwidths is not considered. 
In this work, we consider the application of FD in mmWave indoor wireless systems and we propose joint control of transmission power levels and the beam-level beamwidths of mmWave radios, aiming to maximize energy efficiency. Our solution incorporates the inherit nature of transmission/reception beam-level beamwidths of a mmWave communication link, while employing optimal power control mechanism to maximize the total energy efficiency of the mmWave communication system.

For performance analysis, a realistic Wireless Local Area Network (WLAN) channel model for $60 \mathrm{GHz}$ mmWave systems is considered, where the communicating nodes are randomly deployed in a conference room, examining the NLOS propagations only. We consider three different power budget schemes $(4 \mathrm{dBm}, 10 \mathrm{dBm}, 27 \mathrm{dBm})$ for evaluating the average total energy efficiency performance with respect to system parameters, such as maximum transmit power levels, SI cancellation level and pilot transmission overhead. Numerical results show that the proposed FD mmWave scheme with joint control of transmit power levels and beam-level beamwidths significantly outperforms the FD mmWave system with power control only, Smart HD and traditional HD. The energy efficiency of Smart HD is improved by up to around four times, and FD mmWave is improved by around $30 \%$, considering practical self-interference cancellation levels achieved for 60 $\mathrm{GHz}$ mmWave communications.

The rest of this paper is organized as follows: In Section II, the system model of the FD mmWave indoor wireless communication system is presented, also providing the computations of Signal-to-Interference and Noise Ratio (SINR), achievable transmission rate, and energy efficiency. In Section III, the energy efficiency optimization problem is formulated first for the FD case, for obtaining joint solution of optimal transmit power levels and beam-level beamwidths, then for the HD case for obtaining the joint optimal transmission slot and beamlevel beamwidth solution. Section IV provides performance analysis results and Section V presents our conclusions.

\section{System Model: FD Millimeter WaVe COMMUNICATION}

As shown in Figure 1, we consider a three-node FD mmWave system, such as a wireless local area network or a small cell network, operating at $60 \mathrm{GHz}$ communication band. Node 1, being the system's coordinator is acting as the access point (AP) or base station (BS), while nodes 2 and 3 are the stations (STAs) or user equipments (UEs) of the system. Node 2 is connected to Node 1 in the downlink, while Node 3 is connected to Node 1 in the uplink. Node 1 is receiving from Node 3 and transmitting its data to Node 2 . We consider HD and FD modes of operation, where in FD mode, Node 1 is simultaneously receiving and transmitting, while in all cases Node 2 and Node 3 operate only in HD mode. It is also assumed that for a specific time instant, the channel conditions and the network's topology do not change, i.e. the model assumes quasi-static channel state information (CSI). We let $l$ denote the Non Line-Of-Sight (NLOS) link between
Node 3 and Node 1 , and $m$ for Node 1 and Node 2 NLOS link, as shown in Figure 1.

For realizing the channel between the communicating nodes, assuming there is no LOS link between the nodes, we consider NLOS large scale fading model for the 802.11ad mmWave communication in the conference room environment [19], [20]:

$F L_{a, b}^{c}[d B]=\underbrace{A_{N L O S}+20 \log _{10}\left(f_{c}\right)+10 n_{N L O S} \log _{10}\left(d_{a, b}\right)}_{\text {mean path loss }}+\underbrace{X_{\sigma_{S F_{d B}}}}_{\text {shadowing }}$

where $F L_{a, b}^{c}[\mathrm{~dB}]$ denotes the large scale fading between a transmitter node $a$ and a receiver node $b$ in $\mathrm{dB}, A_{N L O S}$, and $n_{N L O S}$ are scenario and antenna-specific parameters, $f_{c}$ is the system carrier frequency (in $\mathrm{GHz}$ ). $d_{a, b}$ is the distance between the transmitter, and receiver in meters and $X_{\sigma_{S F_{d B}}}$ $\sim \mathcal{N}\left(0, \sigma_{S F_{d B}}\right)$. For the $S T A \leftrightarrow S T A$ communications, $A_{N L O S}=51.5 \mathrm{~dB}, n_{N L O S}=0.6$ and shadow fading standard deviation $\left(\sigma_{S F_{d B}}\right)=3.3 \mathrm{~dB}$. While for $A P \leftrightarrow S T A$ communications, the respective values are $45.5 \mathrm{~dB}, 1.4$ and 3 dB [19], [20].

The model for antenna gain calculations is the well-known sectored antenna model [21], where the transmitting antenna gain is given as;

$$
G_{a, b}^{t}=\left\{\begin{array}{cc}
\frac{2 \pi-\left(2 \pi-\varphi_{a}^{t}\right) z}{\varphi_{a}^{t}}, & \text { if }\left|\theta_{a, b}^{t}\right| \leq \frac{\varphi_{a}^{t}}{2} . \\
z, & \text { otherwise }
\end{array}\right.
$$

For the receiver, the gain is obtained as

$$
G_{a, b}^{r}=\left\{\begin{array}{cc}
\frac{2 \pi-\left(2 \pi-\varphi_{b}^{r}\right) z}{\varphi_{b}^{r}}, & \text { if }\left|\theta_{a, b}^{r}\right| \leq \frac{\varphi_{b}^{r}}{2}, \\
z, & \text { otherwise }
\end{array}\right.
$$

where $G_{a, b}^{t}$ and $G_{a, b}^{r}$ represent the antenna gains of the transmitter $a$ and the receiver $b, \varphi_{a}^{t}$ and $\varphi_{b}^{r}$ denote the beam-level beamwidths, $\theta_{a, b}^{t}$ and $\theta_{a, b}^{r}$ represent the angular differences of the transmitter and receiver with respective to the boresight line, and $z$ is the side lobe level gain having a range of $0 \leq z \ll 1$.

We consider the beam searching overhead alignment delay in the effective transmission rate calculations as in [16].

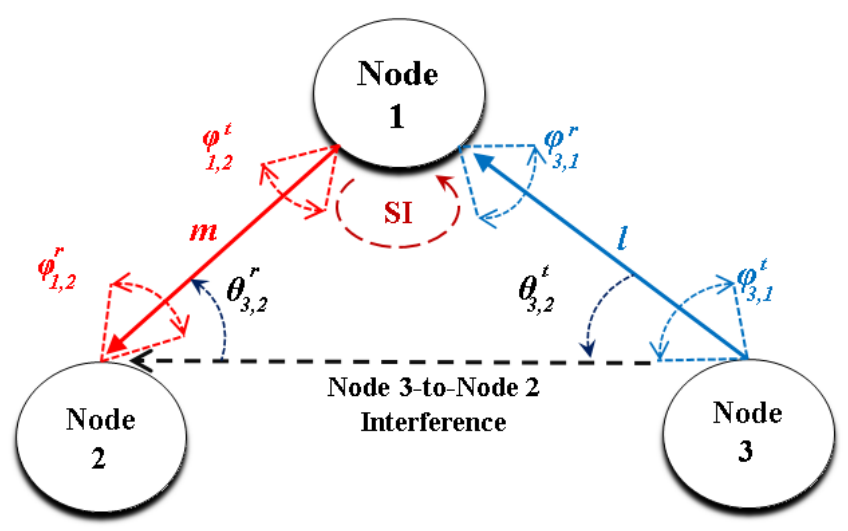

Figure 1: Full duplex mmWave indoor wireless system. 
Letting $T_{p}$ denote the pilot signal transmission duration and $\psi_{a}^{t}, \varphi_{a}^{t}$ and $\psi_{b}^{r}, \varphi_{b}^{r}$ denote the sector-level and beam-level beamwidths of the transmitter $a$ and the receiver $b$ respectively, the alignment delay (continuous approximation) can be calculated as [16],

$$
\tau_{a, b}\left(\varphi_{a}^{t}, \varphi_{b}^{r}\right)=\frac{\psi_{a}^{t} \psi_{b}^{r}}{\varphi_{a}^{t} \varphi_{b}^{r}} T_{p} .
$$

Furthermore, since $\tau_{a, b}$ cannot surpass the total duration for transmission $(T)$, a lower bound on effective beam-level beamwidths can be obtained as,

$$
\varphi_{a}^{t} \varphi_{b}^{r} \geq \frac{T_{p}}{T} \psi_{a}^{t} \psi_{b}^{r}
$$

As the beam-level beamwidths are aligned within the sectorlevels, the upper bounds on the beam-level beamwidths can be settled as $\varphi_{a}^{t} \leq \psi_{a}^{t}$ and $\varphi_{b}^{r} \leq \psi_{b}^{r}$. After the beamlevel beamwidth alignments, the remaining time available for the effective transmission is $T-\tau_{a, b}$ seconds, which can be normalized by the total duration for transmission $T$, to obtain the term $\left(1-\frac{\tau_{a, b}}{T}\right)$ [15], [16].

In the following, achievable rate and energy efficiency metrics are calculated for FD and HD modes for the considered scenario.

\section{A. Achievable Rate}

1) Full duplex case: When Node 1 is operating in FD mode, it will experience some residual SI due to its own transmission, since SI cannot be completely cancelled. The SINR of the link $l$ observed at node 1 can be expressed as:

$$
\Gamma_{1}^{f d}=\frac{P_{3}^{t} G_{3,1}^{t} G_{3,1}^{c} G_{3,1}^{r}}{\gamma_{1}+N_{0} B},
$$

where $P_{3}^{t}$ denotes the transmit power of Node $3, G_{3,1}^{t}$ denotes the transmit antenna gain of Node 3 towards Node $1, G^{c}$ denote the channel gains between the respective nodes obtained as $f\left(F L_{a, b}^{c}\right) . G_{3,1}^{r}$ is the receive antenna gain of Node 1 towards Node $3, N_{0}$ is the noise power density and $B$ is the system's bandwidth. Moreover, $\gamma_{1}$ represents the residual self-interference at the node 1 obtained as, $\gamma_{1}=P_{1}^{t} / \beta$, where $\beta$ denotes SI suppression level for Node 1 [22].

The SINR of the link $m$ observed at Node 2 is calculated as:

$$
\Gamma_{2}^{f d}=\frac{P_{1}^{t} G_{1,2}^{t} G_{1,2}^{c} G_{1,2}^{r}}{P_{3}^{t} G_{3,2}^{t} G_{3,2}^{c} G_{3,2}^{r}+N_{0} B} .
$$

At this point, it is important to note that for the aboveformulated SINR calculations (7), we have assumed the model as defined in [23], where the interfering signal from the Node 3 is provisioned by considering the side lobe level gains (i.e. $G_{3,2}^{t}$ and $G_{3,2}^{r}$ ) [21].

The achievable rates of the links $l$ and $m$ are then calculated as,

$$
\begin{aligned}
& R_{3,1}^{f d}=\left(1-\frac{\tau_{3,1}}{T}\right) B \log _{2}\left(1+\Gamma_{1}^{f d}\right) . \\
& R_{1,2}^{f d}=\left(1-\frac{\tau_{1,2}}{T}\right) B \log _{2}\left(1+\Gamma_{2}^{f d}\right) .
\end{aligned}
$$

The total achievable rate (throughput) of the FD mmWave system is then computed as:

$$
R_{\text {total }}^{f d}=R_{3,1}^{f d}+R_{1,2}^{f d} .
$$

2) Half duplex case: In HD mode, Node 1's transmission and reception operations are time division duplexed, so there is no residual SI. The HD-SNR at Node 1 can be expressed as,

$$
\Gamma_{1}^{h d}=\frac{P_{3}^{t} G_{3,1}^{t} G_{3,1}^{c} G_{3,1}^{r}}{N_{0} B} .
$$

HD-SNR at Node 2 is,

$$
\Gamma_{2}^{h d}=\frac{P_{1}^{t} G_{1,2}^{t} G_{1,2}^{c} G_{1,2}^{r}}{N_{0} B} .
$$

Given $\delta_{3}$, as the fraction of transmission slot designated for Node 3 , the achievable transmission rate of the link $l$ is calculated as [24]:

$$
R_{3,1}^{h d}=\delta_{3}\left(1-\frac{\tau_{3,1}}{T}\right) B \log _{2}\left(1+\Gamma_{1}^{h d}\right) .
$$

For the link $m$,

$$
R_{1,2}^{h d}=\left(1-\delta_{3}\right)\left(1-\frac{\tau_{1,2}}{T}\right) B \log _{2}\left(1+\Gamma_{2}^{h d}\right) .
$$

Therefore, the total transmission rate of mmWave system for the HD case is [24]:

$$
R_{\text {total }}^{h d}=R_{3,1}^{h d}+R_{1,2}^{h d}
$$

\section{B. Energy Efficiency}

The consumed power per node is calculated by modeling power consumption realistically at each node of the FD mmWave system according to [7]:

$$
P_{i}=\frac{P_{i}^{t}}{\omega}+\epsilon R+P_{\text {circuit }, \text { static }}+\zeta P_{A I C},
$$

where $P_{i}^{t}$ is the transmit power level of Node $i, \omega$ is the amplifier's drain efficiency, $\epsilon$ is the power consumed with respect to the unit achievable transmission rate [25], $R$ is the achievable transmission rate, $P_{\text {circuit, static }}$ is the circuit's static power consumption, $P_{A I C}$ represents the power consumed for analog SI cancellation, which is usually larger than $50 \mathrm{~mW}$ [26], and in this paper we assumed a constant consumption for SI cancellation of $50 \mathrm{~mW} . \zeta$ is an indicator variable, which is 1 for FD and 0 for HD support. The power consumption at all the nodes are calculated via (16), except for Node 2. Since the node is only HD-capable and acts only as the receiver, the first and the last terms of the equation (16), i.e. $\frac{P_{i}^{t}}{\omega}$ and $P_{A I C}$ are not included. The total power consumption of all the nodes of the FD mmWave system can then be expressed as:

$$
\text { Power }_{\text {total }}=P_{1}+P_{2}+P_{3} \text {. }
$$

Energy efficiency is a well-known performance metric for the system-level evaluation of a communication system, defined as the ratio of the achievable transmission rate to the power consumed [18]. Mathematically, 


$$
\eta=\frac{\text { Achievable transmission rate }}{\text { Power consumed }}(\text { bits/joule }) .
$$

\section{Resource Allocation For ENERGy EFFICIENCY}

In the FD case, in order to maximize the total energy efficiency $\eta^{f d}$ of the entire FD mmWave system, we propose to jointly control the transmit power levels and beam-level beamwidths of all the nodes of the network. For this purpose, we formulate the following optimization problem:

$$
\begin{aligned}
& \max _{P_{i}^{t}, \varphi^{t}, \varphi^{r}} \eta^{f d}, \\
0 & \leq P_{i}^{t} \leq P_{\max }, \forall i, \\
\varphi_{i}^{t} & \leq \psi_{i}^{t}, \forall i, \\
\varphi_{i}^{r} & \leq \psi_{i}^{r}, \forall i, \\
\psi_{i}^{t} \psi_{i}^{r} \frac{T_{p}}{T} & \leq \varphi_{i}^{t} \varphi_{i}^{r}, \forall i .
\end{aligned}
$$

Here, $i \in\{1,2,3\}$ represents the nodes of the system as specified in Figure 1. Since this problem, (19) is non-convex with non-linear constraints, it is difficult to find the exact solution analytically. Therefore, interior-point algorithm [27] is implemented in MATLAB to obtain a numerical solution.

In the HD case, for maximizing the total energy efficiency $\eta^{\text {hd }}$ of the entire HD mmWave system, we consider joint control of the transmission slot interval and beam-level beamwidths of all the nodes, which results in the following optimization problem:

$$
\begin{aligned}
& \max _{\delta, \varphi^{t}, \varphi^{r}} \eta^{h d}, \\
& 0 \leq \delta_{i} \leq 1, \forall i, \\
& \text { s.t. } \quad \leq \psi_{i}^{t}, \forall i, \\
& \varphi_{i}^{t} \leq \psi_{i}^{r}, \forall i, \\
& \psi_{i}^{t} \psi_{i}^{r} \frac{T_{p}}{T} \leq \varphi_{i}^{t} \varphi_{i}^{r}, \forall i .
\end{aligned}
$$

The optimization problem in (20) is also non-convex with non-linear constraints, so again a numerical solution [27] is found.

\section{Numerical Results}

In this section, we present the energy efficiency analysis of FD mmWave communication system with the proposed joint power and beam-level beamwidth optimization, in comparison to the following schemes: 1) FD with power control only [18] having a fixed beam-level beamwidth of 15.4 degrees, which is an acceptable beam-level beamwidth of mmWave system transceivers [16], 2) Smart HD with joint transmission slot control and beam-level beamwidth, as discussed in Section III, and 3) HD with transmission slot control only, with a fixed beam-level beamwidth of 15.4 degrees [16]. Also, $\psi=$

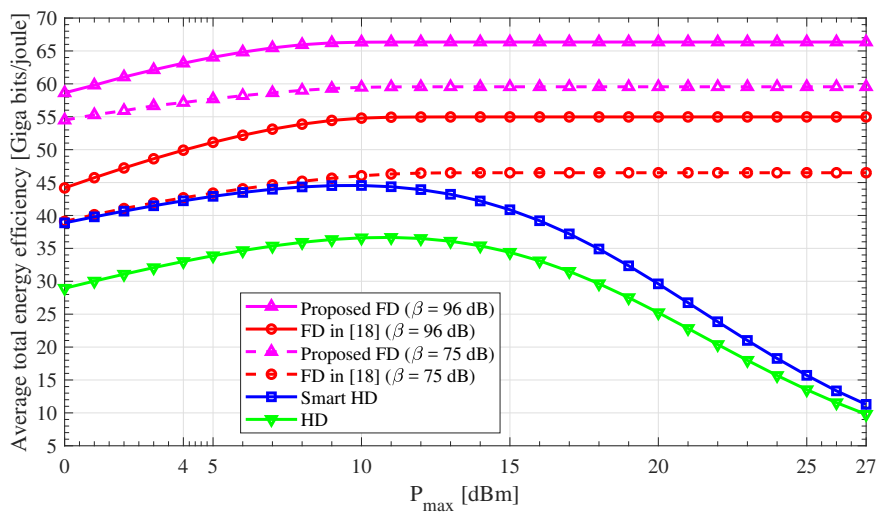

Figure 2: Average total energy efficiency vs. $P_{\max }[\mathrm{dBm}]$.

90 degrees for all transmitters and receivers and $\mathrm{z}=0.05$ [16]. We consider the 802.11ad compliant mmWave indoor WLAN scenario as depicted in Figure 1 in a $3 \mathrm{~m}$ x $4.5 \mathrm{~m}$ x $3 \mathrm{~m}$ (W x L $\mathrm{x} \mathrm{H})$ conference room, where Node 1 is located at the center of the ceiling, while Nodes 2 and 3 are randomly-deployed in the room [20]. By performing Monte Carlo simulations, we assess the average total energy efficiency performance over 200 random topologies, as a function of maximum transmit power level, SI cancellation level and pilot transmission overhead. The simulation parameters are set as follows (unless otherwise stated): $B=3 \mathrm{GHz}, N_{0}=-174 \mathrm{dBm} / \mathrm{Hz}, \omega=0.25, \epsilon=5$ $\mathrm{mW}$ per Gb/s, $P_{\text {circuit, static }}=200 \mathrm{~mW}$, and $P_{A I C}=50 \mathrm{~mW}$, and $\frac{T_{p}}{T}=10^{-4}[16],[18]$.

Figure 2 depicts the impact of the maximum transmit power level $\left(P_{\max }\right)$ on the average total energy efficiency of the mmWave system. Evidently, the proposed FD at both the observed SI cancellation levels, i.e. $\beta=75 \mathrm{~dB}$ and $\beta=96$ $\mathrm{dB}$ provided the highest total energy efficiency for all the considered maximum power transmit levels. For $P_{\max }=4$ $\mathrm{dBm}$, the proposed FD scheme with $\beta=75 \mathrm{~dB}$ provided a net average total energy efficiency gain of $33.92 \%$ over FD with power control only [18]. From $P_{\max }=0$ to $13 \mathrm{dBm}$, for $\beta=75 \mathrm{~dB}$, there is no considerable advantage in employing FD with power control only [18] over Smart HD. For the highest considered maximum transmit power level i.e. $P_{\max }$ $=27 \mathrm{dBm}$, the proposed FD scheme with $\beta=75 \mathrm{~dB}$ showed an improvement in the average total energy efficiency of about 4.2x against Smart HD, whereas no significant improvement was offered by the proposed FD scheme against the FD with power control only [18].

Figure 3 shows the effect of SI cancellation $(\beta)$ on the average total energy efficiency of the mmWave system, for $\frac{T_{p}}{T}=10^{-4}$. Three different power budgets are considered, Figure 3(a) representing low power budget $\left(P_{\max }=4 \mathrm{dBm}\right)$ i.e. WPANs [16], Figure 3(b) for moderate power budget $\left(P_{\max }=10 \mathrm{dBm}\right)$, which is the maximum power transmit limit for $60 \mathrm{GHz}$ mmWave communications in Japan [28]-[30], and Figure 3(c) for high power budget $\left(P_{\max }=27 \mathrm{dBm}\right)$, which is the maximum transmit power limit for $60 \mathrm{GHz}$ mmWave communications in USA [28]-[30]. Figure 3(a) clearly depicts 
that the proposed FD scheme with the low power budget [16] readily outperformed the traditional $\mathrm{HD}$ even at $\beta=35 \mathrm{~dB}$, while the FD having power control only [18] has its HD cross-over point at about $\beta=63 \mathrm{~dB}$. Furthermore, it can be observed that the proposed FD scheme required the SI cancellation of at least $\beta=55 \mathrm{~dB}$ in order to surpass the average total energy performance of Smart HD. Considering the practical SI cancellation level i.e. $\beta=75 \mathrm{~dB}$, the proposed FD scheme showed better performance than FD with power control only [18] and Smart HD, which could be improved further with higher SI cancellation levels. Figure 3(b) shows that the proposed FD scheme with the moderate power budget [28]-[30] still outperformed the traditional HD even at $\beta=35$ $\mathrm{dB}$. Moreover, it can be seen that as the maximum transmit power level (i.e. $P_{\max }$ ) is increased from $4 \mathrm{dBm}$ to $10 \mathrm{dBm}$, the minimum SI cancellation level required by the proposed FD scheme to outperform Smart HD has been shifted from 55 $\mathrm{dB}$ (Figure 3(a)) to $52 \mathrm{~dB}$. Figure 3(c) provides the efficacy of employing the high power budget [28]-[30] for assessing the average total energy efficiency of the mmWave system. Interestingly, the proposed FD scheme with the high power budget (i.e. $27 \mathrm{dBm}$ ) outperformed all the benchmark schemes. This is due to the fact that in both the Smart HD and the traditional HD schemes, there are no power control mechanisms. Consequently, upon increasing the maximum transmit power level (i.e. up to about $9 \mathrm{dBm}$ ), the total achievable throughput increased up to a certain extent, after which the imposed power consumption become dominate, causing a decline in the average total energy efficiency. Note that, our results presented in this paper assume constant power consumption for SI cancellation. However, higher SI cancellation could cost higher power, creating a trade-off between $\beta$ and energy efficiency, which is left as a future study.

Figure 4 highlights the impact of the pilot transmission overhead $\left(\frac{T_{p}}{T}\right)$ on the average total energy efficiency of the mmWave system for the three different power budgets as in Figure 3. Two SI-cancellation levels, $\beta=75 \mathrm{~dB}$ and $96 \mathrm{~dB}$, are specifically considered for analyzing the FD schemes as the former is the practically achieved SI cancellation in [11] and the latter is the ideal maximum SI cancellation required for $60 \mathrm{GHz}$ mmWave communication [11]. Figure 4(a) shows the impact of $\frac{T_{p}}{T}$ on the average total energy efficiency of the mmWave system for $P_{\max }=4 \mathrm{dBm}$ [16]. As the overhead is increased from $10^{-4}$ to $10^{-2}$, the average total energy performance gain of the proposed FD scheme $(\beta=75 \mathrm{~dB})$ over Smart HD downgraded from $34.98 \%$ to $29.93 \%$. Figure 4(b) provides the effect of $\frac{T_{p}}{T}$ on the average total energy efficiency of the mmWave system for $P_{\max }=10 \mathrm{dBm}$ [28]-[30]. As the overhead rose from $10^{-4}$ to $10^{-2}$, the performance gain of the proposed FD scheme $(\beta=75 \mathrm{~dB})$ against the Smart HD scheme declined from $33.46 \%$ to $23.85 \%$. In comparison to the FD with power control only [18], the performance gain of the proposed FD scheme downgraded from $29.2 \%$ to $8.7 \%$. Figure 4(c) highlights the implications of $\frac{T_{p}}{T}$ on the average total energy efficiency of the mmWave system for $P_{\max }=27$ $\mathrm{dBm}$ [28]-[30]. Interestingly, it can be seen that by increasing the overhead from $10^{-4}$ to $10^{-2}$, although the performance of the proposed scheme $(\beta=75 \mathrm{~dB})$ is decreased by $39 \%$, still the scheme provided a net gain in the average total energy efficiency of about 3.5x over Smart HD.

\section{CONCLUSIONS}

In this paper, we have proposed joint control of transmission power and beam-level beamwidths for applying FD in mmWave indoor wireless systems. The proposed FD scheme is analyzed by evaluating the average total energy efficiency for an indoor wireless communication scenario, considering IEEE 802.11ad model. By considering the three distinct power budgets, our results show the improvement potential of the proposed FD scheme, as it outperformed Smart HD by up to 4.2x and FD with power control only by up to $33.92 \%$ for $75 \mathrm{~dB}$ practically-attained SI cancellation. Our results show that mmWave FD with our proposed solution promises a good potential for green $5 \mathrm{G}$ networks. Incorporation of the proposed solution into a MAC protocol and analysis for a larger network is our future work.

\section{REFERENCES}

[1] T. S. Rappaport, S. Sun, R. Mayzus, H. Zhao, Y. Azar, K. Wang, G. N. Wong, J. K. Schulz, M. Samimi, and F. Gutierrez, "Millimeter wave mobile communications for $5 \mathrm{~g}$ cellular: It will work!," IEEE Access, vol. 1, pp. 335-349, 2013.

[2] A. I. Sulyman, A. Alwarafy, G. R. MacCartney, T. S. Rappaport, and A. Alsanie, "Directional radio propagation path loss models for millimeter-wave wireless networks in the 28-, 60-, and 73-ghz bands," IEEE Transactions on Wireless Communications, vol. 15, pp. 69396947, Oct 2016.

[3] S. Singh, F. Ziliotto, U. Madhow, E. Belding, and M. Rodwell, "Blockage and directivity in $60 \mathrm{ghz}$ wireless personal area networks: from cross-layer model to multihop mac design," IEEE Journal on Selected Areas in Communications, vol. 27, pp. 1400-1413, October 2009.

[4] S. Rangan, T. S. Rappaport, and E. Erkip, "Millimeter-wave cellular wireless networks: Potentials and challenges," Proceedings of the IEEE, vol. 102, pp. 366-385, March 2014.

[5] "Ieee 802.15.3c: Standard for information technology- local and metropolitan area networks- specific requirements- part 15.3: Amendment 2: Millimeter-wave-based alternative physical layer extension," 2009.

[6] "Ieee 802.11ad. part 11: Wireless lan medium access control (mac) and physical layer (phy) specifications amendment 3: Enhancements for very high throughput in the $60 \mathrm{ghz}$ band," 2012.

[7] Z. Wei, X. Zhu, S. Sun, Y. Huang, A. Al-Tahmeesschi, and Y. Jiang, "Energy-efficiency of millimeter-wave full-duplex relaying systems: Challenges and solutions," IEEE Access, vol. 4, pp. 4848-4860, 2016.

[8] A. Ghosh, T. A. Thomas, M. C. Cudak, R. Ratasuk, P. Moorut, F. W. Vook, T. S. Rappaport, G. R. MacCartney, S. Sun, and S. Nie, "Millimeter-wave enhanced local area systems: A high-data-rate approach for future wireless networks," IEEE Journal on Selected Areas in Communications, vol. 32, pp. 1152-1163, June 2014.

[9] A. Sabharwal, P. Schniter, D. Guo, D. W. Bliss, S. Rangarajan, and R. Wichman, "In-band full-duplex wireless: Challenges and opportunities," IEEE Journal on Selected Areas in Communications, vol. 32, pp. 1637-1652, Sept 2014.

[10] M. Duarte and A. Sabharwal, "Full-duplex wireless communications using off-the-shelf radios: Feasibility and first results," in 2010 Conference Record of the Forty Fourth Asilomar Conference on Signals, Systems and Computers, pp. 1558-1562, Nov 2010.

[11] T. Dinc, A. Chakrabarti, and H. Krishnaswamy, "A $60 \mathrm{ghz}$ cmos fullduplex transceiver and link with polarization-based antenna and rf cancellation," IEEE Journal of Solid-State Circuits, vol. 51, pp. 11251140, May 2016.

[12] E. Everett, A. Sahai, and A. Sabharwal, "Passive self-interference suppression for full-duplex infrastructure nodes," IEEE Transactions on Wireless Communications, vol. 13, pp. 680-694, February 2014. 


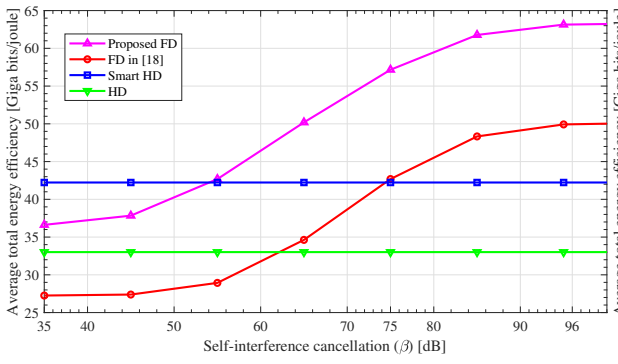

(a)

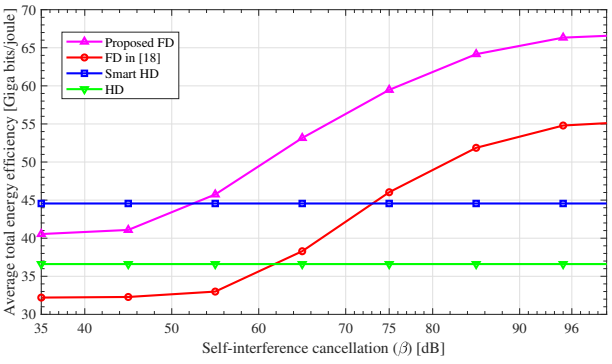

(b)

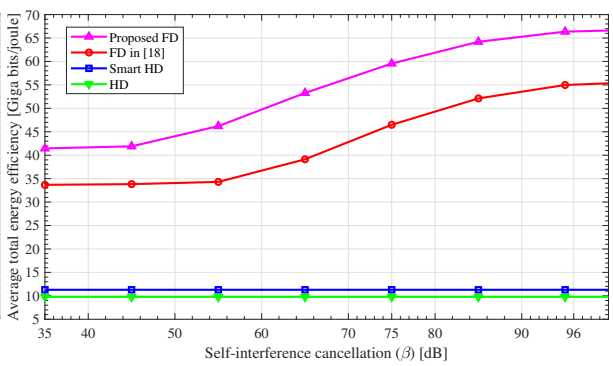

(c)

Figure 3: Average total energy efficiency vs. self-interference cancellation $(\beta), \frac{T_{p}}{T}=10^{-4}$ : (a) $P_{\max }=4 \mathrm{dBm}$ (WPANs [16]), (b) $P_{\max }=10 \mathrm{dBm}$ (Japan) [28]-[30], and (c) $P_{\max }=27 \mathrm{dBm}$ (USA) [28]-[30].

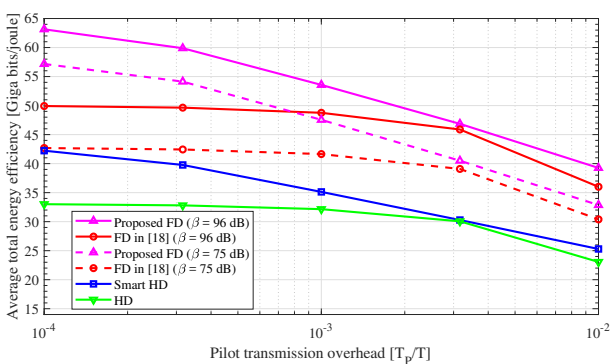

(a)

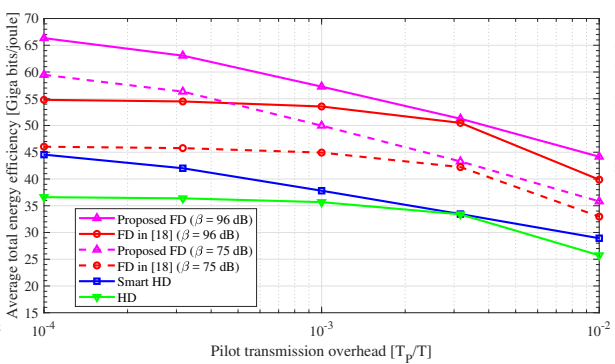

(b)

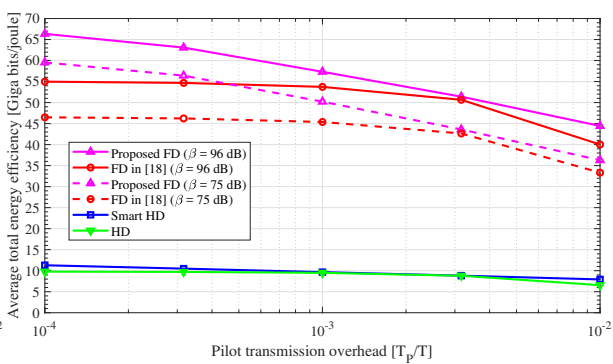

(c)

Figure 4: Average total energy efficiency vs. practical pilot transmission overhead $\left(\frac{T_{p}}{T}\right)$ [16]: (a) $P_{\max }=4 \mathrm{dBm}$ (WPANs [16]), (b) $P_{\max }=10 \mathrm{dBm}$ (Japan) [28]-[30], and (c) $P_{\max }=27 \mathrm{dBm}$ (USA) [28]-[30].

[13] M. Duarte, A. Sabharwal, V. Aggarwal, R. Jana, K. K. Ramakrishnan, C. W. Rice, and N. K. Shankaranarayanan, "Design and characterization of a full-duplex multiantenna system for wifi networks," IEEE Transactions on Vehicular Technology, vol. 63, pp. 1160-1177, March 2014.

[14] C. L. I, C. Rowell, S. Han, Z. Xu, G. Li, and Z. Pan, "Toward green and soft: a $5 \mathrm{~g}$ perspective," IEEE Communications Magazine, vol. 52, pp. 66-73, February 2014.

[15] C. Perfecto, J. D. Ser, M. I. Ashraf, M. N. Bilbao, and M. Bennis, "Beamwidth optimization in millimeter wave small cell networks with relay nodes: A swarm intelligence approach," in European Wireless 2016; 22th European Wireless Conference, pp. 1-6, May 2016.

[16] H. Shokri-Ghadikolaei, L. Gkatzikis, and C. Fischione, "Beam-searching and transmission scheduling in millimeter wave communications," in 2015 IEEE International Conference on Communications (ICC), pp. 1292-1297, June 2015.

[17] L. Li, K. Josiam, and R. Taori, "Feasibility study on full-duplex wireless millimeter-wave systems," in 2014 IEEE International Conference on Acoustics, Speech and Signal Processing (ICASSP), pp. 2769-2773, May 2014.

[18] Z. Wei, X. Zhu, S. Sun, Y. Huang, T. Ma, and Y. Jiang, "Energy efficiency optimization for full-duplex relaying with hybrid self-interference cancellation in $60 \mathrm{ghz}$ indoor wireless systems," in 2015 IEEE/CIC International Conference on Communications in China (ICCC), pp. 1-6, Nov 2015.

[19] T. S. Rappaport, Y. Xing, G. R. MacCartney, A. F. Molisch, E. Mellios, and J. Zhang, "Overview of millimeter wave communications for fifthgeneration $(5 \mathrm{~g})$ wireless networkswith a focus on propagation models," IEEE Transactions on Antennas and Propagation, vol. 65, pp. 62136230, Dec 2017.

[20] A. Maltsev, V. Erceg, E. Perahia, C. Hansen, R. Maslennikov, A. Lomayev, A. Sevastyanov, A. Khoryaev, G. Morozov, M. Jacob, S. Priebe, T. Krner, S. Kato, H. Sawada, K. Sato, and H. Harada, "Channel models for $60 \mathrm{ghz}$ wlan systems," doc.: IEEE 802.11-09/0334r8, May 2010.

[21] J. Wildman, P. H. J. Nardelli, M. Latva-aho, and S. Weber, "On the joint impact of beamwidth and orientation error on throughput in directional wireless poisson networks," IEEE Transactions on Wireless Communications, vol. 13, pp. 7072-7085, Dec 2014.

[22] S. Goyal, P. Liu, S. Panwar, R. A. DiFazio, R. Yang, J. Li, and E. Bala, "Improving small cell capacity with common-carrier full duplex radios," in 2014 IEEE International Conference on Communications (ICC), pp. 4987-4993, June 2014.

[23] P. Gupta and P. R. Kumar, "The capacity of wireless networks," IEEE Transactions on Information Theory, vol. 46, pp. 388-404, Mar 2000.

[24] K. Akcapinar and O. Gurbuz, "Full-duplex bidirectional communication under self-interference," in 2015 13th International Conference on Telecommunications (ConTEL), pp. 1-7, July 2015.

[25] C. Xiong, G. Y. Li, S. Zhang, Y. Chen, and S. Xu, "Energy- and spectralefficiency tradeoff in downlink ofdma networks," IEEE Transactions on Wireless Communications, vol. 10, pp. 3874-3886, November 2011.

[26] C. Marcu, D. Chowdhury, C. Thakkar, J. D. Park, L. K. Kong, M. Tabesh, Y. Wang, B. Afshar, A. Gupta, A. Arbabian, S. Gambini, R. Zamani, E. Alon, and A. M. Niknejad, "A $90 \mathrm{~nm}$ cmos low-power $60 \mathrm{ghz}$ transceiver with integrated baseband circuitry," IEEE Journal of Solid-State Circuits, vol. 44, pp. 3434-3447, Dec 2009.

[27] A. Wchter and L. T. Biegler, "On the implementation of an interiorpoint filter line-search algorithm for large-scale nonlinear programming," Mathematical Programming, pp. 25-57, April 2006.

[28] S. Chairman, "60 ghz kick-off of the analysis version 1," CEPT-ECC SRD-MG, vol. SRDMG(17)043, pp. 1-21, FEbruary 2017.

[29] K. Sakaguchi, E. M. Mohamed, H. Kusano, M. Mizukami, S. Miyamoto, R. E. Rezagah, K. Takinami, K. Takahashi, N. Shirakata, H. Peng, T. Yamamoto, and S. Nanba, "Millimeter-wave wireless lan and its extension toward 5g heterogeneous networks," IEICE Transactions on Communications, vol. E98.B, no. 10, pp. 1932-1948, 2015.

[30] N. Guo, R. C. Qiu, S. S. Mo, and K. Takahashi, "60-ghz millimeterwave radio: Principle, technology, and new results," EURASIP Journal on Wireless Communications and Networking, vol. 2007, p. 068253, Dec 2006. 